\title{
Chronic active hepatitis induced by Pazopanib mimicking hypervascular liver metastases in a patient with recurrent soft tissue sarcoma: A case report
}

\author{
ANA EZPONDA ${ }^{1}$, IGNACIO GONZÁLEZ DE LA HUEBRA ${ }^{1}$, \\ MARTA CALVO $^{1}$, MIGUEL ÁNGEL IDOATE ${ }^{2}$ and ISABEL VIVAS ${ }^{1}$ \\ Departments of ${ }^{1}$ Radiology and ${ }^{2}$ Pathology, Clínica Universidad de Navarra, Pamplona 31008, Spain
}

Received December 22, 2017; Accepted June 13, 2018

DOI: 10.3892/ol.2018.9114

\begin{abstract}
Pazopanib is the first multitargeted tyrosine-kinase inhibitor approved for the treatment of patients with advanced non-adipocytic soft tissue sarcoma (STS). It has been demonstrated to improve progression-free survival without impairing health-associated quality of life. However, Pazopanib is associated with several adverse side effects associated with inhibition of the vascular endothelial growth factor receptor. These include hepatotoxicity, as manifested by abnormal liver function tests. To the best of our knowledge, the current study presents the first case of a patient with recurrent STS who developed biopsy proven Pazopanib-induced chronic active hepatitis and whose previous computed tomography examination demonstrated multiple hypervascular liver lesions. These lesions were indistinguishable from metastases and to the best of our knowledge, have not been described previously. These lesions therefore appear to be a novel finding of Pazopanib-induced chronic active hepatitis. It is crucial to be aware of this unusual finding within a clinical setting, to avoid overstaging and early discontinuation of effective treatment.
\end{abstract}

\section{Introduction}

Soft tissue sarcomas (STS) account for $1 \%$ of all malignant adult tumors and this rare mesenchymal neoplasm comprises around 50 histological types (1).

Correspondence to: Dr Isabel Vivas, Department of Radiology, Clínica Universidad de Navarra, 36 Avenida Pío XII, Pamplona 31008, Spain

E-mail: isvivasp@unav.es

Abbreviations: STS, soft tissue sarcoma; TKIS, tyrosine-kinase inhibitors; VEGFR, vascular endothelial growth factor receptor; MR, magnetic resonance; DWI, diffusion weighted imaging; ADC, apparent diffusion coefficient

Key words: Pazopanib, tyrosine kinase inhibitors, toxicity, chronic active hepatitis, soft tissue sarcoma, metastases, hypervascular lesions
According to evidence, among 20-30\% of all patients with STS will have local recurrence of the tumor, which can be effectively treated with surgery and radiotherapy (2). The conventional first-line treatment for advanced STS includes anthracycline and trabectedin, in addition to radiation therapy $(3,4)$.

However, patients with advanced STS have a poor prognosis with median overall survival of approximately 12 months from diagnosis and, until recently, only doxorubicin and ifosfamide had shown consistent efficacy against this group of sarcomas (3). Fortunately, the improved understanding of the molecular basis of this cancer has led to the development of targeted therapies, which have revolutionized the approach to the treatment of sarcomas $(1,5,6)$.

Angiogenesis has been identified as a crucial factor for tumor growth and metastases and represents an important target for controling cancer progression. Nevertheless, the existence of multiple angiogenic signaling pathways, with drivers such as the vascular endothelial growth factor (VEGF) and platelet derived growth factor (PDGF), makes it imperative to identify the specific vascularization profile of each tumor $(6,7)$.

Several studies have confirmed that angiogenesis plays an important role in the pathogenesis of STS. The presence of high levels of VEGF expression is associated with a higher malignancy grade in advanced STS and with worse metastasis-free and overall survival rates in localized disease (8). Therefore, the molecular mediators of angiogenesis represent a group of potential targets for the treatment of STS.

Pazopanib is an oral multitargeted tyrosine-kinase inhibitor (TKI) active against VEGF receptors (VEGFR-1, VEGFR-2, VEGFR-3), PDGF receptors (PDGFR- $\alpha$, PDGFR- $\beta$ ) and c-kit (9).

Several studies have explored the activity of pazopanib against STS. In a phase II study, Sleijfer et al demonstrated the antitumor activity of pazopanib and its safety profile in patients with advanced STS (9). On the basis of this publication, the PALETTE study, a randomized phase III trial designed by the Soft Tissue and Bone Sarcoma Group of the European Organization for Research and Treatment of Cancer (STCSG-EORTC), analyzed the efficacy of pazopanib in STS. Pazopanib increased progression-free survival by a median 
of 3 months compared with placebo, thus becoming an oral therapeutic option after prior chemotherapy in non-adipocytic STS (10-12).

Based on the results of PALETTE study, in 2012 the FDA approved Pazopanib for the management of advanced STS (excluding adipocytic STS and GIST). This agent was the first anti-angiogenic drug approved for this group of sarcomas.

Nevertheless, Pazopanib is associated with several side effects related to the inhibition of VEGFR. Abnormalities in liver function tests have been observed in patients treated with this drug in different clinical trials, with increases in the levels of serum transaminases and bilirubin, although imaging findings have been normal (13).

In the present study, we report a case of chronic active hepatitis associated with Pazopanib use in a patient with recurrent STS whose liver function tests were normal. To the best of our knowledge, the present study reports a new imaging finding of Pazopanib induced chronic active hepatitis secondary to treatment with Pazopanib in a patient with recurrent STS and normal liver function tests.

\section{Case report}

A 76-year-old woman diagnosed with undifferentiated pleomorphic sarcoma on the right forearm was admitted in 2009 to the Medical Oncology Department of our center. She was treated initially with surgery and brachytherapy.

Subsequently, she presented multiple regional recurrences and axillary lymph node metastases. She was treated with surgery, radiotherapy and chemotherapy (liposomal Adriamycin and Trabectedin). In February 2017 the patient presented a recurrence in the middle region of the forearm, with poorly defined infiltration of the anterior musculature. In the rest of the thoracoabdominal examination, no images suggesting the spread of the disease were found. The oncologist decided to start treatment with Pazopanib (800 mg administered daily). The patient had no risk factors for viral hepatitis, history of alcohol abuse and she was not taking potentially hepatotoxic medications.

Two months after the beginning of the treatment, a computed tomography (CT) scan was performed to assess tumor response and showed that the patient's disease was now stable.

In June 2017, the patient returned to the Oncology Department for a routine medical examination. The patient was asymptomatic and her physical examination was unremarkable. Her laboratory tests including liver function tests remained normal. At that time, in addition to Pazopanib, her medications included an anti-platelet agent due to her history of stroke, folic acid and vitamin D. A re-staging abdominal CT scan was performed.

The contrast-enhanced abdominal CT scan revealed multiple hypervascular hepatic lesions, not visible in the portal phase, suggestive of metastatic disease (Fig. 1).

An abdominal MR with liver-specific contrast (Gadoxetate disodium, Primovist ${ }^{\circledR}$; Bayer Schering Pharma, Berlin, Germany) was performed confirming the presence of multiple hypervascular lesions. These nodular lesions were hyperintense on T2WI, with intense enhancement during the arterial-phase and became hypointense in the hepatocyte phase. The lesions showed restriction of diffusion with increased DWI signal and ADC reduction (Figs. 2 and 3). All were consistent with metastatic disease.

Given the suspicion of metastatic disease in a patient with recurrent sarcoma, a liver biopsy was performed. The pathology report revealed the presence of a moderately inflammatory infiltrate of the portal spaces composed of lymphoplasmacytic cells and a significant number of eosinophils, which caused distortion of the hepatic architecture. It associated edema and necrosis in punches, with occasional Councilman bodies. There was no evidence of malignancy (Fig. 4).

With the definitive anatomopathological diagnosis of chronic active hepatitis induced by Pazopanib and due to the complete absence of clinical and analytical repercussions, it was decided to continue the course of Pazopanib.

During follow-up, the patient remained asymptomatic and liver function tests were repeated with normal results (data not shown). An abdominal CT scan performed three months later revealed a significant decrease in size of the liver lesions (Fig. 5).

Our Institution's ethics committee (Clínica Universidad de Navarra, Pamplona, Spain) approved the study (IRB approval no 2018/056) and the patient signed the informed consent form.

\section{Discussion}

In the present study, the case of a female patient with recurrent STS who developed hepatic hypervascular nodular lesions as an atypical manifestation of chronic hepatitis induced by Pazopanib is described.

Pazopanib is an oral multi-targeted TKI active against VEGFR with high efficacy in the treatment of recurrent STS. Unfortunately, this drug is not free from side effects. The EORTC (study no. 62043) investigated the toxicity induced by Pazopanib and reported that the most common symptoms were fatigue, diarrhea, appetite loss and nausea/vomiting $(11,14,15)$. The major adverse effects were cardiovascular (especially hypertension), skin reactions, gastrointestinal and hepatic disorders, mild myelosuppression and proteinuria. Other less frequent side effects of Pazopanib in patients with STS include hypothyroidism, venous and arterial thrombotic events, pneumothorax, arrhythmias and cardiac dysfunction $(12,14,16)$.

Among these adverse effects, transient elevations in serum aminotransferases are also associated with Pazopanib and may occur in up to $50 \%$ of patients. Most of these elevations occur within the first 18 weeks of treatment. In different clinical trials, increased aminotransferase levels $>5$ times the upper limit of normal (ULN) occurred in $8 \%$ of patients (13). Increases in bilirubin levels are less frequent and are usually associated with fatal cases of clinically apparent acute liver injury. Kapadia et al reported that $7-9 \%$ of their patients developed grade 3 or 4 liver toxicity, with one case of fatal hepatotoxicity in their series (17).

Klempner et al published two cases of high-grade hepatotoxicity associated with Pazopanib in patients with metastatic renal cell carcinoma with accompanying liver-biopsy information (13). The pathological specimen was characterized by the presence of an infiltrate of lymphocytes, histiocytes, neutrophils and eosinophils within the portal tracts. Mild 

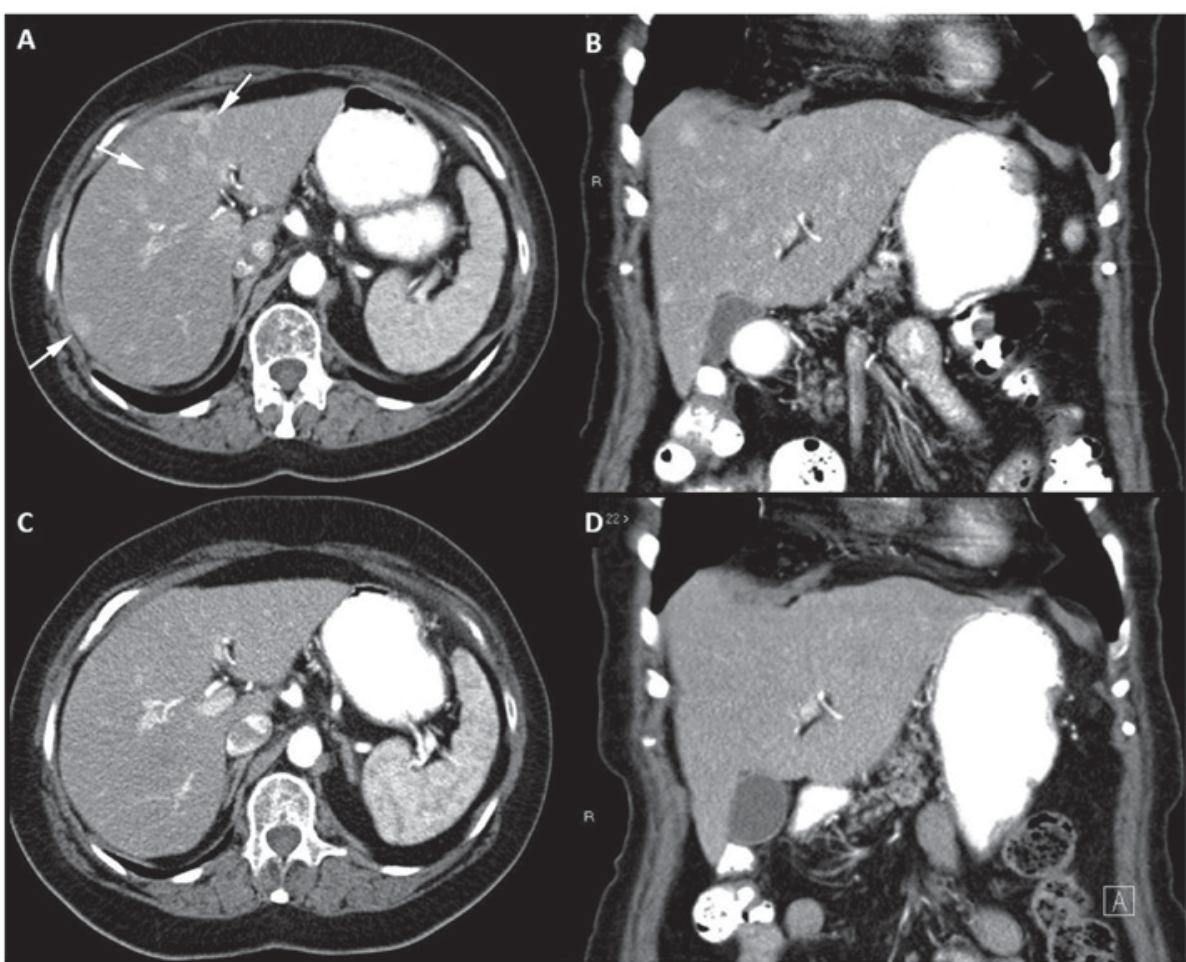

Figure 1. Serial contrast-enhanced CT imaging of a patient with recurrent soft tissue sarcoma in treatment with pazopanib. Contrast-enhanced abdominal CT performed in June 2017. Multiple hypervascular hepatic lesions are identified in the late arterial phase in the (A) axial and (B) coronal planes in both lobes of the liver (three are shown with arrows). Baseline contrast-enhanced abdominal CT performed in April 2017 reveals a normal liver without hepatic lesions in the (C) axial and (D) coronal planes. CT, computed tomography.

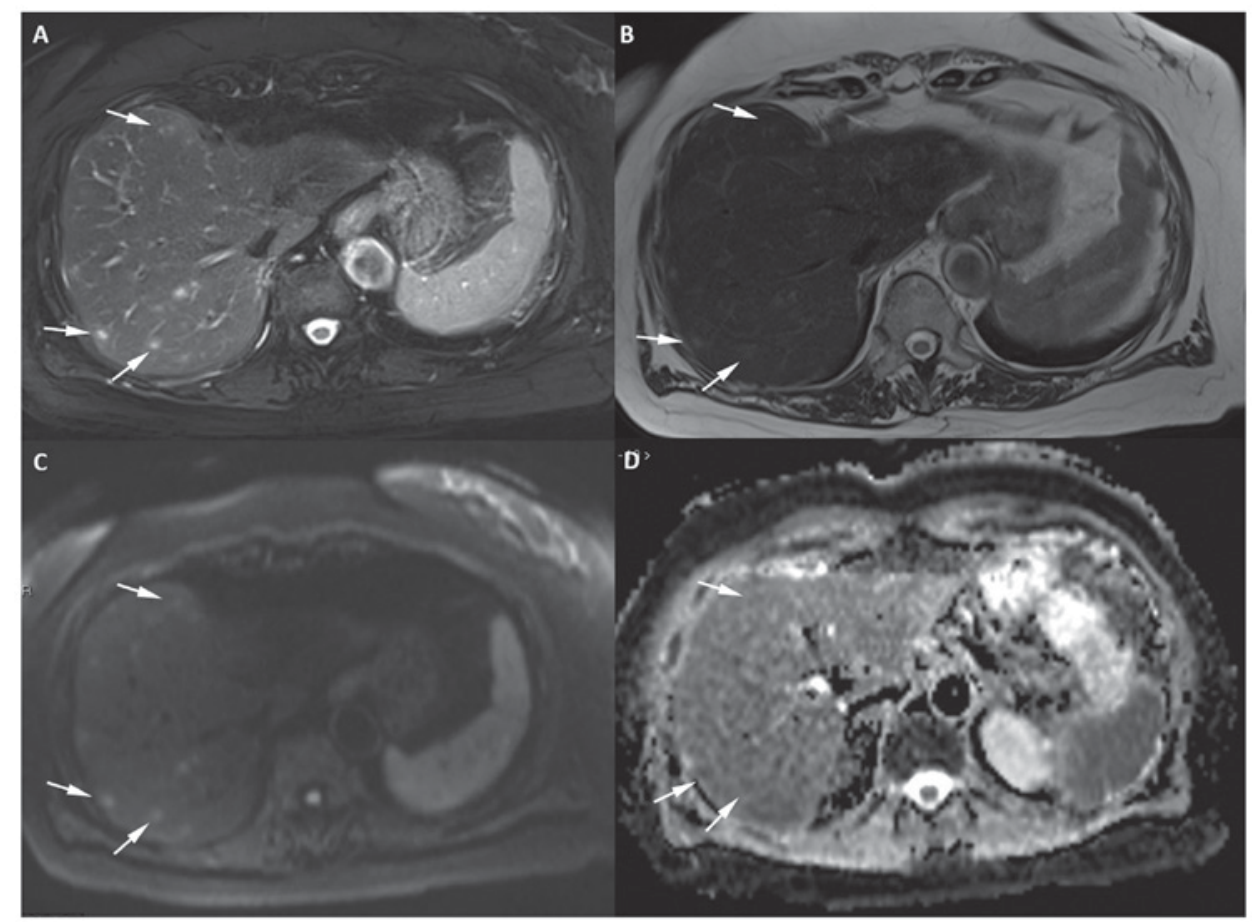

Figure 2. Axial 1.5 T MR image of the liver with 5-mm section thickness in a patient with recurrent soft tissue sarcoma receiving treatment with Pazopanib. The image reveals a minimum of 8 focal lesions in the right lobe of the liver (three of the lesions are marked with white arrows). (A) The lesions show very high signal intensity on the T2-weighted fat-suppressed sequence and (B) mild hyperintensity on the T2-weighted sequence when compared with the background liver. (C) The lesions show high signal on DWI at $b=800 \mathrm{smm}^{-2}$ and (D) mild restriction on the apparent diffusion coefficient map. DWI, diffusion weighted imaging; MR, magnetic resonance.

centrilobular intrahepatic cholestasis was also present because of bile duct damage.
The classical radiologic manifestation of this increase in these liver test parameters, if present, is a decrease in hepatic 


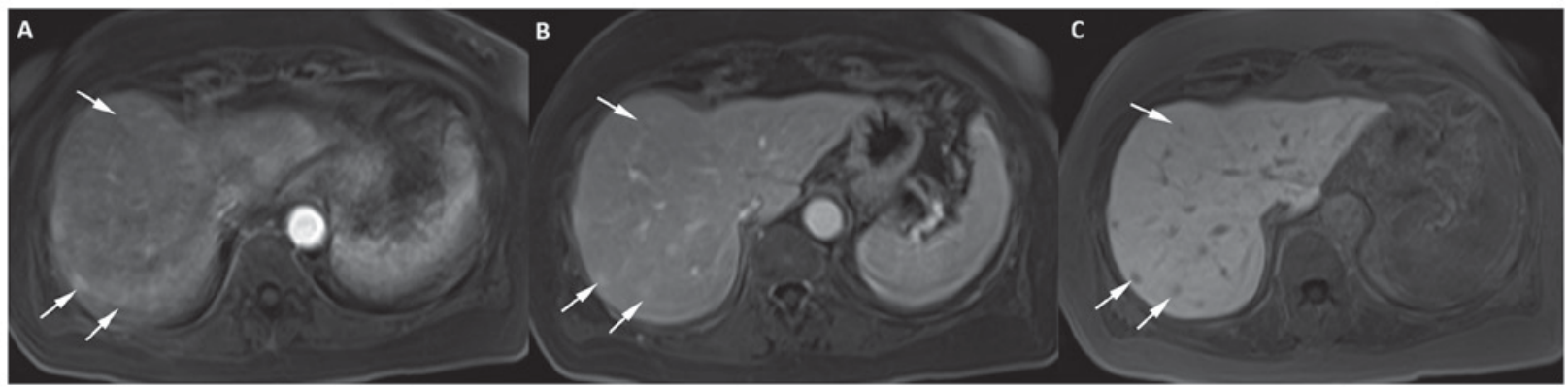

Figure 3. Multiple hepatic nodules mimicking metastases in a patient with recurrent soft tissue sarcoma under treatment with Pazopanib. Abdominal 1.5-T MR axial images with hepatospecific contrast. Axial fat-suppressed T1-weighted 1,5T MR images of the liver with 3-mm section thickness at (A) 30 sec and (B) $20 \mathrm{~min}$ post intravenous administration of Gd-EOB-DTPA $(0.1 \mathrm{ml} / \mathrm{kg}$ body weight) indicate multiple focal lesions in both lobes of the liver (three are marked with white arrows). (A) The lesions indicate heterogenous hypervascularity in the arterial phase, (B) with persisting mild hypervascularity in the portal phase and (C) low signal intensity compared with the surrounding liver parenchyma in the hepatospecific phase. MR, magnetic resonance.

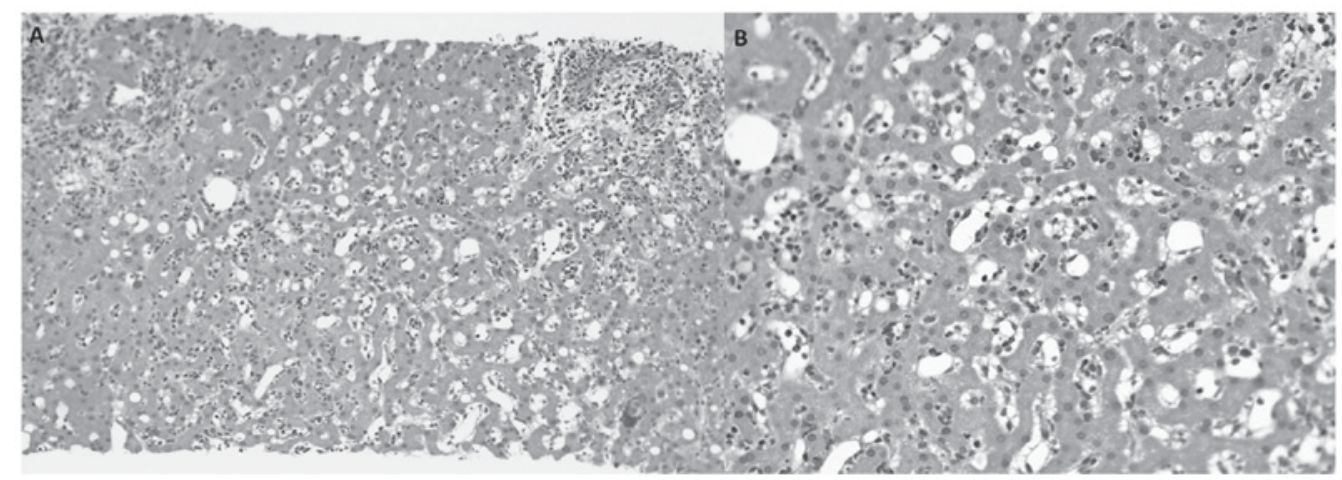

Figure 4. Microscopic observation of the liver specimen with hematoxylin and eosin staining following the biopsy of several hepatic nodules. (A) Inflammatory portal infiltration with preservation of the limiting plate, sinusoidal dilatation and slight macrogotular steatosis are observed (magnification, x100). (B) Lobular lymphoeosinophilic inflammatory infiltration with sinusoidal dilatation is present. Liver trabeculation is preserved. There is no evidence of malignancy (magnification, $\mathrm{x} 200$ ).

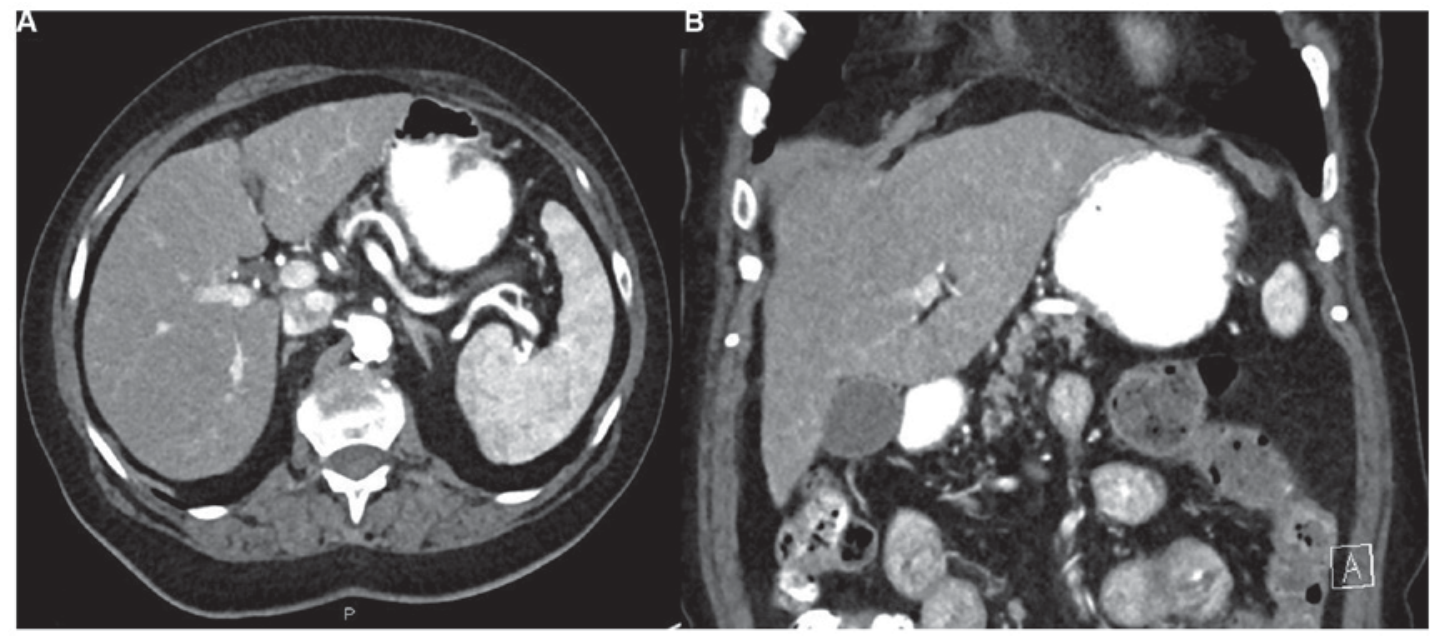

Figure 5. Follow-up abdominal computed tomography scan performed in October 2017. The images were obtained 35 sec post administration of intravenous contrast material. The liver lesions indicate a significant decrease in size in the (A) axial and (B) coronal planes.

attenuation, similar to steatosis (18). Additional findings of hepatitis are periportal edema, mild hepatomegaly and enlarged periportal lymph nodes.

Although it is thought hepatotoxicity may be related to the hepatic metabolism of Pazopanib (through the CYP3A4 pathway), the underlying mechanism remains unclear (13).
According to guidelines, before and during treatment, liver function test results should be monitored every 4 weeks, at least for the first 4 months of therapy $(13,14)$. These guidelines state that isolated increases in serum aminotransferase levels over 8 times the ULN should lead to temporary cessation of Pazopanib until levels return to $\leq 3$ times the ULN. 
When increases are $>3$ times the ULN and and are accompanied by increases in bilirubin $>2$ times the ULN, Pazopanib should be permanently discontinued $(14,17)$. In our case, given the absence of any clinical and analytical impact, it was decided to continue treatment with Pazopanib.

Our patient, who was undergoing treatment with Pazopanib, did not present any abnormalities in her liver tests although new multiple focal liver lesions were observed. In this clinical scenario of recurrent STS, the first diagnosis to be considered when hypervascular liver lesions appear de novo in a CT scan is metastatic disease.

The differential diagnosis should include other possibilities such as focal nodular hyperplasias, regenerative nodular hyperplasias or, rarely, foci of peliosis. With the aim of better characterizing our patient's state, an abdominal MR scan with hepatospecific contrast was performed. Both focal nodular hyperplasia and regenerative hyperplasia should show contrast elimination in the hepatocyte phase. In our patient, the nodules were hypointense in this phase, so hyperplasias were discarded. The peliosis, corresponding to vascular cavities, should not restrict the diffusion or show hyperintensity on T2WI, as the nodules showed in our case. Another diagnosis could be atypical arterioportal shunts that may manifest as nodular enhancement in the arterial-phase with slightly hyperintense nodules on T2WI. However, in the hepatocyte phase these lesions are usually hyper or isointense $(19,20)$.

Fibrous pseudotumors due to autoimmune chronic hepatitis are not enhanced in the hepatocyte-phase due to the lack of functioning hepatocytes, but they are not enhanced either during the arterial or portal phase (19).

According to these imaging findings, metastases represented the most likely diagnosis. Due to the absence of local progression and the achievement of stable disease as established in the previous CT scan, it was decided to perform an ultrasound guided liver biopsy. The biopsy of several hepatic nodular lesions showed chronic active hepatitis that involved portal spaces with an inflammatory infiltrate composed of lymphocytes and numerous eosinophils with no malignant cells. Overall, the biopsy results were consistent with drug-induced hepatotoxicity four months after pazopanib initiation.

Based on an extensive literature review, this is the first case reporting the extremely rare manifestation of chronic active hepatitis induced by Pazopanib. In this case, the performance of a liver biopsy was decisive for accurately diagnosing the inflammatory process. It is thus crucial for both radiologists and oncologists, to be aware of this unusual radiological manifestation of hepatitis induced by Pazopanib in order to not confuse the hepatitis for metastases and to avoid overstaging and early discontinuation of an effective treatment.

\section{Acknowledgements}

Not applicable.

\section{Funding}

No funding was received.

\section{Availability of data and materials}

Data sharing is not applicable to this article, as no datasets were generated or analyzed during the current study.

\section{Authors' contributions}

$\mathrm{AE}$ and IV designed the study and wrote the first draft of the manuscript. AE, IV, IGH, MC and MAI editing the manuscript. $\mathrm{AE}$ and IV provided resources and reviewed the manuscript. $\mathrm{AE}, \mathrm{IGH}$ and MC performed data analysis. MAI performed the anatomopathological examination. IV supervised the study and was responsible for ensuring that questions related to the accuracy or integrity of any part of the study were appropriately investigated and resolved.

\section{Ethics approval and consent to participate}

The Ethics Committee of the Clínica Universidad de Navarra approved the present study (IRB approval no. 2018/056) and the patient provided written informed consent.

\section{Patient consent for publication}

The patient provided written informed consent for the publication of their data and associated images.

\section{Competing interests}

The authors declare that they have no competing interests.

\section{References}

1. Clark MA, Fisher C, Judson I and Thomas JM: Soft-tissue sarcomas in adults. N Engl J Med 353: 701-711, 2005.

2. Zagars GK, Mullen JR and Pollack A: Malignant fibrous histiocytoma: Outcome and prognostic factors following conservation surgery and radiotherapy. Int J Radiat Oncol Biol Phys 34: 983-994, 1996.

3. Le Cesne A, Blay JY, Judson I, Van Oosterom A, Verweij J, Radford J, Lorigan P, Rodenhuis S, Ray-Coquard I, Bonvalot S, et al: Phase II study of ET-743 in advanced soft tissue sarcomas: a European Organitation for the Research and Treatment of Cancer (EORTC) soft tissue and bone sarcoma group trial. J Clin Oncol 23: 576-584, 2005.

4. Demetri GD, Chawla SP, von Mehren M, Ritch P, Baker LH, Blay JY, Hande KR, Keohan ML, Samuels BL, Schuetze S, et al: Efficacy and safety of trabectedin in patients with advanced or metastatic liposarcoma or leiomyosarcoma after failure of prior anthracyclines and ifosfamide: Results of a randomized phase II study of two different schedules. J Clin Oncol 27: 4188-4196, 2009.

5. Weis SM and Cheresh DA: Tumor angiogenesis: Molecular pathways and therapeutic targets. Nat Med 17: 1359-1370, 2011.

6. DuBois S and Demetri G: Markers of angiogenesis and clinical features in patients with sarcoma. Cancer 109: 813-819, 2007.

7. Sleijfer S, van der Graaf WT and Blay JY: Angiogenesis inhibition in non-GIST soft tissue sarcomas. Oncologist 13: 1193-1200, 2008.

8. Chao C, Al-Saleem T, Brooks JJ, Rogatko A, Kraybill WG and Eisenberg B: Vascular endothelial growth factor and soft tissue sarcomas: Tumor expression correlates with grade. Ann Surg Oncol 8: 260-267, 2001

9. Sleijfer S, Ray-Coquard I, Papai Z, Le Cesne A, Scurr M, Schöffski P, Collin F, Pandite L, Marreaud S, De Brauwer A, et al: Pazopanib, a multikinase angiogenesis inhibitor, in patients with relapsed or refractory advanced soft tissue sarcoma: A phase II study from the European organisation for research and treatment of cancer-soft tissue and bone sarcoma group (EORTC study 62043). J Clin Oncol 27: 3126-3132, 2009. 
10. van der Graaf WT, Blay JY, Chawla SP, Kim DW, Bui-Nguyen B, Casali PG, Schöffski P, Aglietta M, Staddon AP, Beppy Y, et al: Pazopanib for metastatic soft-tissue sarcoma (PALETTE): A randomised, double-blind, placebo-controlled phase 3 trial. Lancet 379: 1879-1886, 2012.

11. Kasper B, Sleijfer S, Litière S, Marreaud S, Verweij J, Hodge RA, Bauer S, Kerst JM and van der Graaf WT: Long-term responders and survivors on pazopanib for advanced soft tissue sarcomas: Subanalysis of two European Organisation for Research and Treatment of Cancer (EORTC) clinical trials 62043 and 62072. Ann Oncol 25: 719-724, 2014.

12. Coens C, van der Graaf WT, Blay JY, Chawla SP, Judson I, Sanfilippo R, Manson SC, Hodge RA, Marreaud S, Prins JB, et al: Health-related quality-of- life results from PALETTE: A randomized, double-blind, phase 3 trial of pazopanib versus placebo in patients with soft tissue sarcoma whose disease has progressed during or after prior chemotherapy-a European Organization for Research and Treatment of Cancer-Soft Tissue and Bone Sarcoma Group Global Network Study (EORTC 62072). Cancer 121: 2933-2941, 2015.

13. Klempner SJ, Choueiri TK, Yee E, Doyle LA, Schuppan D and Atkins MB: Severe pazopanib-induced hepatotoxicity: Clinical and histologic course in two patients. J Clin Oncol 30: e264-e286, 2012.

14. GlaxoSmithKline: Votrient (pazopanib) tablets prescribing information. http://www.accessdata.fda.gov/drugsatfda docs/label/2009/022465lbl.pdf
15. Santoni M, Conti A, De Giorgi U IacovelliR, Pantano F, Burattini L, Muzzonigro G, Berardi R, Santini D and Cascinu: Risk of gastrointestinal events with sorafenib, sunitinib and pazopanib in patients with solid tumors: A systematic review and meta-analysis of clinical trials. Int J Cancer, 135: 763-773, 2014.

16. Chen HX and Cleck JN: Adverse effects of anticancer agents that target the VEGF pathway. Nat Rev Clin Oncol 6: 465-477, 2009.

17. Kapadia S, Hapani S, Choueiri TK and Wu S: Risk of liver toxicity with the angiogenesis inhibitor pazopanib in cancer patients. Acta Oncol 52: 1202-1212, 2013.

18. Klapko O, Ghoulam E, Jakate S, Eswaram S and Usha L: Anastrozole-induced autoimmune hepatitis: A rare complication of breast cancer therapy. Anticancer research 37: 4173-4176, 2017.

19. Vilgrain V, Lagadec $M$ and Ronot $M$ : Pitfalls in Liver Imaging 2016. Radiology; 278: 34-51, 2016.

20. Silva AC, Evans JM, McCullough AE, Jatoi MA, Vargas HE and Hara AK: MR Imaging of hypervascular liver masses: a review of current techniques. Radiographics 29: 385-402, 2009. 